\title{
Aprende a Experimentar en Física sobre un Entorno Virtual 3.0.
}

\section{José Daniel Sierra Murillo}

a Universidad de La Rioja, Departamento de Química, Área de Física Aplicada; Complejo

Científico-Tecnológico, C/ Madre de Dios 51 26006-Logroño, daniel.sierra@unirioja.es.

\begin{abstract}
The objective of this Teaching Innovation Project, "Learn to Experiment in Physics on a Virtual Environment 3.0", is to generate audiovisual resources that allow teachers and students to enjoy physical phenomena permanently. Besides the improvement of the learning of the Physics, it is of great interest the acquisition on the part of the student of competences on autonomous and collaborative work, as well as of computer skills, fundamental in the science and technology of the 21st century.
\end{abstract}

\section{Keywords:}

Experiment in Physics, Virtual Environment 3.0, audiovisual resources, competences on autonomous and collaborative work, computer skills, 21st century.

\section{Resumen}

El objetivo de este Proyecto de Innovación Docente, "Aprende a Experimentar en Física sobre un Entorno Virtual 3.0”, es generar recursos audiovisuales que permitan a profesores y alumnos disfrutar de los fenómenos físicos de forma permanente. Además de la mejora del aprendizaje de la Física, es de gran interés la adquisición por parte del alumno de competencias sobre trabajo autónomo y colaborativo, así como de habilidades informáticas, fundamentales en la ciencia y tecnología del siglo XXI.

\section{Palabras clave:}

Experimentar en Física, Entorno Virtual 3.0, recursos audiovisuales, competencias sobre trabajo autónomo y colaborativo, habilidades informáticas, siglo XXI.

\section{Introducción}

Las demostraciones experimentales de Física están asociadas al desarrollo de la ciencia moderna y fueron utilizadas desde el siglo XVII en sociedades científicas, academias, salones y conferencias para todo tipo de público. También han sido empleadas de forma generalizada como herramientas docentes en instituciones educativas y universidades 
constituyéndose como prácticas docentes habituales en diferentes países. A pesar de que su uso está recogido explícitamente en el proyecto europeo “Tuning” (González y Wagenaar, 2003) de Física para la armonización de titulaciones universitarias, en nuestro país no forma parte de las prácticas docentes generalizadas. A este respecto, en la Facultad de Ciencias Físicas de la Universitat de València existe un proyecto docente que ha incorporado el uso de las demostraciones experimentales como herramienta habitual en sus clases, y que dicho proyecto ha obtenido resultados altamente satisfactorios (Ferrer 2012).

Algunos de los estudiantes que llegan a la universidad no han cursado Física durante la enseñanza secundaria y, la mayoría de quienes sí lo han hecho, no han realizado prácticas de laboratorio o visto demostraciones experimentales. Por lo que, por lo general, desconocen las causas/consecuencias experimentales de los fenómenos físicos más básicos. Por ello, el uso de demostraciones experimentales permite articular un proceso de observación racional en el que se emitan conclusiones en términos de los modelos físicos estudiados en el ámbito teórico, algo esencial en la comprensión del método científico; además de ofrecer la posibilidad de involucrar de forma activa a los alumnos en su propio proceso de aprendizaje. (Aparicio, F., González, R.M. y Sobrevila, M.A., 2005) (Calvo, I., Lopez-Guede, J.M. y Zulueta, E., 2010) (Alba, J., Torregrosa, C. y Del Rey, R., 2015).

Como hemos descrito previamente, la propia naturaleza intrínseca de las demostraciones experimentales hace que sea posible involucrar a los alumnos de forma activa y en primera persona en el proceso de aprendizaje. Esto permite pasar de un planteamiento centrado en el profesor a uno más orientado al estudiante.

En otro orden de cosas, la educación superior ha experimentado un gran cambio dentro del sistema educativo de la sociedad actual en diferentes ámbitos (Ortega, 2004; González, 2008) como, por ejemplo:

- La evolución de los procesos de formación desde entornos presenciales convencionales hacia otros ámbitos más amplios.

- La demanda generalizada para que los estudiantes adquieran competencias específicas y transversales (Bolonia, 2009), así como ciertas habilidades necesarias para su aprendizaje continuo.

Para ello, pueden apoyarse en las nuevas Tecnologías de la sociedad de la Información y de la Comunicación (TIC), sin olvidar hacer énfasis en la docencia. Cambios de estrategias didácticas de los profesores y alumnos para acceder, buscar, compartir y gestionar toda la información referida a diferentes materiales objeto de estudio y aprendizaje, es decir, en los procesos de innovación docente. (Mora Mora H., Signes Pont, M. T., De Miguel Casado, G. y Gilart Iglesias, V., 2015)

En cuanto al EV-3.0, se considera como un estado en el que Internet proporciona condiciones de gran interés para que los individuos y las organizaciones puedan compartir información independientemente de las redes y dispositivos que utilicen. (Mora Mora H., Azorín López, J., Jimeno Morenilla, A., Sánchez Romero, J. L., Pujol López, F., García Rodríguez, J., Serra Pérez, J. A., Morell Giménez, V., Rives Pérez, M. F., Saval Calvo, M., García García, A. y Orts Escolano, S., 2016) Las Webs 3.0 (Figura 1) también se han 
venido a denominar Webs semánticas (Berners-Lee, 2001; W3C, 2001). Este nuevo concepto de Web semántica constituye esencialmente una extensión de la Web tradicional en la que la información se coloca de forma estructurada para permitir su fácil consulta y acceso tanto por interlocutores humanos como por sistemas automáticos (Silva, J. M., Mahfujur, A. S. Md. y El Saddik, A., 2008; Nacer y Aissani, 2014).

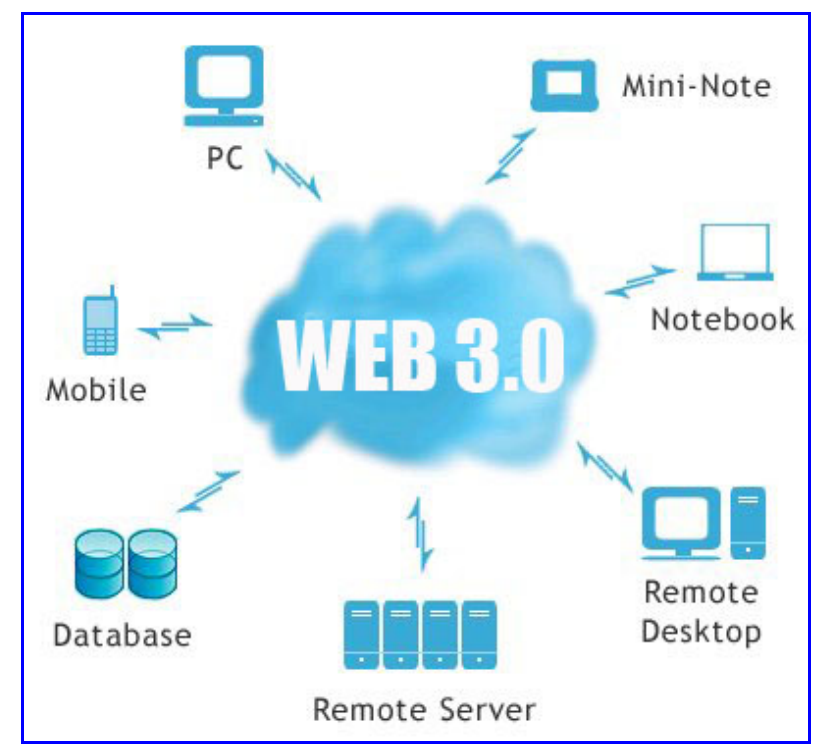

Fig. 1 Visión esquemática de la Web 3.0.

En el ámbito educativo esas herramientas adquieren más relevancia debido a que contribuyen a revisar los planteamientos docentes actuales. Los estudiantes, ya no solo tienen que ser buenos conocedores de cada materia en cuestión, sino también deben desarrollar otras actitudes como la creatividad, el espíritu crítico y la capacidad para el aprendizaje continuo que la sociedad les demanda. Todo ello, en línea con la Declaración Mundial para la Educación Superior en el siglo XXI (Granados, 2011) y la Declaración de Bolonia 2020 (Bolonia, 2009).

Seguidamente se describen brevemente los apartados de los que consta este artículo. A continuación (Sección "Objetivos"), se exponen los objetivos que se pretenden alcanzar a través de este proyecto innovador. Posteriormente, y dentro de la Sección "Desarrollo de la innovación”, se describe el desarrollo de la metodología utilizada en este proyecto mediante una planificación en tres Fases. Por lo que respecta a la Sección titulada "Resultados", en ella se exponen brevemente los logros obtenidos mediante el desarrollo del presente proyecto de innovación. Finalmente, se exhiben unas conclusiones que reafirman el gran valor del uso racional de las TIC en el estudio/aprendizaje Teórico/Experimental de la Física. 


\section{Objetivos}

El principal objetivo de este trabajo es preparar los primeros recursos audiovisuales asociados al Proyecto de Innovación Docente titulado: “Aprende a Experimentar en Física sobre un Entorno Virtual 3.0”, así como poner de manifiesto el carácter multidisciplinar que puede presentar este tipo de herramientas en un EV-3.0.

El desarrollo de estas demostraciones experimentales en formato audiovisual conlleva la generación de nuevos recursos multimedia tales como guías descriptivas, mini-vídeos y memorias que permitan a profesores y estudiantes planificar, grabar, almacenar e intercambiar en un E.V-3.0 las diferentes experiencias sobre diversos fenómenos físicos de forma permanente, más allá del uso instantáneo del material en el aula presencial. Esta colección (o banco audiovisual) se está desarrollando de forma colaborativa con otros profesores que participan o no en el proyecto. Conviene recordar que, aunque de gran interés en el proceso educativo, las metodologías activas basadas en el Aprendizaje Basado en Proyectos (ABP o PBL, Project-Based Learning) suponen una mayor carga/dedicación docente para el profesor (Alba, Torregrosa, del Rey, 2015).

El carácter multidisciplinar de este trabajo presenta importantes ventajas de cara al acercamiento a una temática fundamental (Física) no solo desde la perspectiva de las habituales titulaciones científico-tecnológicas, sino desde otras con un marcado carácter más didáctico/divulgativo.

Otro objetivo no menos importante consiste en conseguir involucrar a más profesores en un futuro cercano y consolidar un grupo de trabajo de innovación docente con aquellos participantes que demuestren un interés para participar activamente en la consecución de este proyecto inicial.

Por otra parte, dado que es un proyecto innovador sobre Experiencias de Física en un EV3.0, otros objetivos fundamentales son la mejora de las competencias del trabajo autónomo y colaborativo del alumno, entre los alumnos de los Grupos de Laboratorio (GL) y de estos con el profesor (Mazur, 1997). Ambas, apoyadas en la Metodología "Flipped Learning" (MFL), (Prieto, 2015) y dentro del EV-3.0. Además de la mejora en el aprendizaje de la Física, es de gran interés la adquisición por parte del alumno de habilidades informáticas, muy importantes en un entorno científico-tecnológico del siglo XXI. (Karpicke and Blunt, 2011; Deslauriers, L., Schelew, E. and Wieman C., 2011; Freeman, S., Eddy, S. L., McDonough, M., Smith M. K., Okoroafor N., Jordt, H. and Wenderoth, M. P., 2014)

\section{Desarrollo de la innovación}

Este proyecto pretende utilizar cualquier plataforma virtual con el objetivo de compatibilizar los trabajos autónomos y colaborativo de los alumnos en la preparación de Experiencias en Física mediante la utilización de un EV-3.0. Este entorno puede ser, desde la Plataforma Virtual de la Universidad de La Rioja (UR) (Figura 2), hasta cualquier sistema de intercambio de información virtual: WhatsApp (foros, grupos, etc.), redes sociales, etc. La innovación reside en utilizar como herramienta de intercambio de 
información multimedia, debate crítico y aprendizaje estos nuevos entornos virtuales que forman parte de los usos y costumbres de la sociedad actual, siglo XXI.

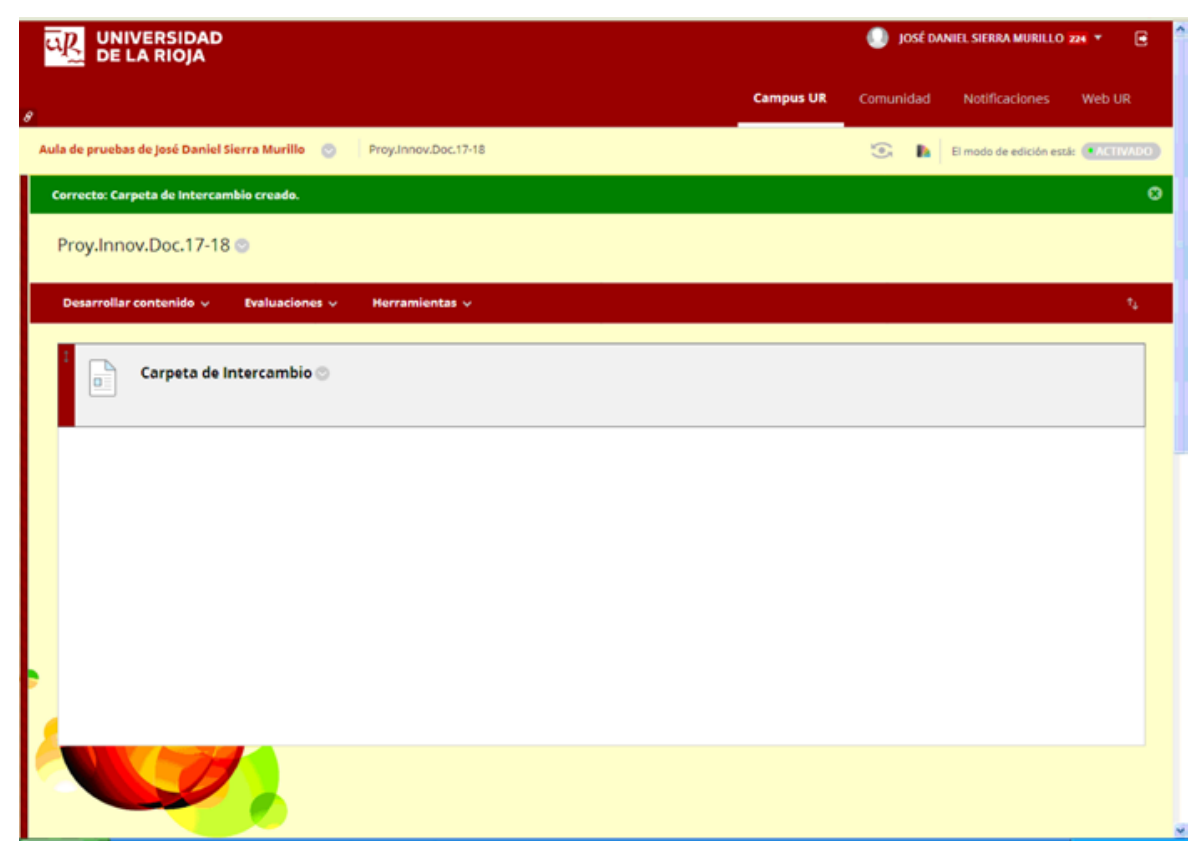

Fig. 2 Plataforma Virtual de la Universidad de La Rioja.

La planificación de la metodología utilizada en este proyecto consta de tres fases.

\section{Primera Fase}

En la primera fase, se pretende evolucionar la enseñanza tradicional (centrada en el protagonismo del profesor que explica todo a sus alumnos) hacia otro tipo de enseñanza en la que el protagonismo se reparta más entre el profesor y sus alumnos. Para que sean estos los que profundicen en el estudio de los temas objeto de investigación teórico-experimental a partir de los materiales y/o referencias proporcionados previamente por el profesor. Todo ello, a través de un trabajo autónomo-colaborativo dentro de un EV-3.0. El trabajo autónomo y colaborativo del alumno en la preparación de Experiencias de Física a través de la MFL y la utilización de un EV-3.0 es sumamente interesante. Por ello, el objetivo fundamental de esta fase es buscar la eficacia autónoma y colaborativa de cada alumno en dicho trabajo de preparación como miembro activo.

Segunda Fase

La optimización de la primera fase, con un posible asesoramiento complementario del profesor, promueve una mejora sustancial en el buen desarrollo posterior de la experimentación (fase 2), que necesita un trabajo constructivo/eficiente de los equipos de alumnos en el desarrollo de las mencionadas experiencias. Por otra parte, su grabación en soporte audiovisual requiere una claridad de ideas sobre el proceso experimental tanto en su realización como en su descripción. (Figura 3) 


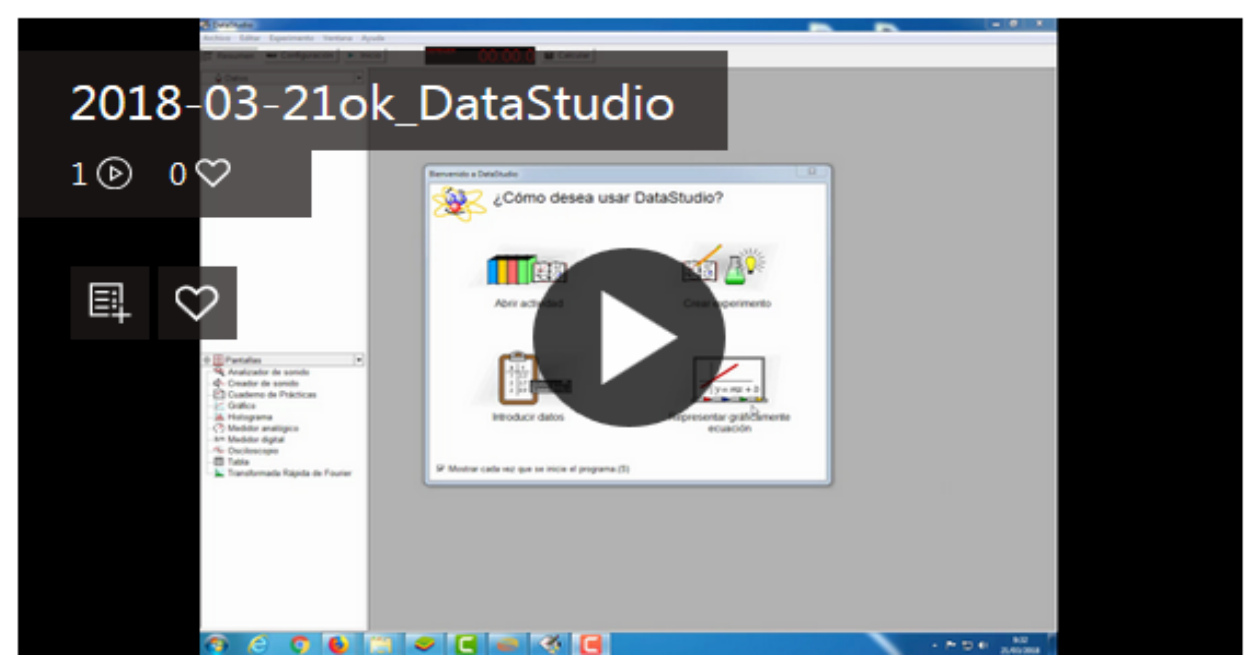

Fig. 3 Carátula del mini-vídeo de una de las Experiencias de Física.

Una vez trabajada con suficiencia las experiencias, y ya dentro del Laboratorio de Física (LF):

a) Comenzará el montaje experimental, para su realización y filmación. Se hará uso de las observaciones anotadas por los alumnos a lo largo del proceso de preparación anteriormente indicado (Fase 1) serán de gran ayuda. Se debe recordar a los alumnos:

- Que es muy importante un correcto montaje del sistema experimental para evitar posibles errores sistemáticos.

- La correcta utilización del hardware y software disponible en el LF con el fin de que consolide unas muy útiles habilidades informáticas necesarias en su posterior dedicación discente/docente, investigadora y profesional en general.

b) Con el sistema experimental correctamente montado, se procederá a la toma de las medidas experimentales y su filmación.

- Hacerles notar a los alumnos la importancia de anotar/(comentar en la filmación) todas las vicisitudes observadas en el transcurso del proceso de medidas: dificultades conceptuales surgidas en el proceso de medida, eventualidades técnicas, etc.. Esta información multimedia compartida en el EV-3.0 mejora el crecimiento consolidado en el aprendizaje científico-tecnológico.

c) Finalmente, se realizará el análisis de las medidas experimentales.

- Recalcar en una buena interpretación física de los resultados de tales análisis, así como en los razonamientos pertinentes que justifiquen el correcto uso del Método Científico sobre el experimento objeto de estudio.

\section{Tercera Fase}

La tercera fase permite la posibilidad de transmitir/compartir la información multimedia obtenida/generada en todo el proceso de aprendizaje a través de los mencionados entornos virtuales crea hábitos de transparencia y superación entre los alumnos. 


\section{Resultados}

Alguno de los resultados que se obtienen con este proyecto son, entre otros, corroborar la importancia de la utilización de las nuevas tecnologías de la sociedad de la información y de la comunicación, no solo en la transmisión de información multimedia, sino también en los procesos de aprendizaje universitario, todo ello, dentro de un EV-3.0. Este perfil del proyecto conjuga con la filosofía de Bolonia 2020 relativas a los cambios en los modelos de docencia-aprendizaje.

Dentro de los resultados perseguidos a lo largo de la puesta en práctica del proyecto de innovación aquí presentado, se exponen a continuación los más significativos:

Se produce una evidente mejoría del trabajo autónomo y colaborativo entre los alumnos, y también entre alumnos-profesor, principalmente a través del EV-3.0.

Además, es significativo el progreso del alumnado en la utilización de:

- La conexión entre plataformas informáticas (hardware y software) con sistemas experimentales donde la adquisición de datos se realiza de forma automática.

- Las plataformas informáticas (hardware y software) para el análisis de los resultados experimentales por parte del alumno.

- Diferentes entornos virtuales utilizados para la transmitir y compartir la información multimedia utilizada y generada en todo el proceso por los mismos alumnos y entre alumnos-profesor.

* Un resultado sumamente importante es la creación de un banco de información multimedia del que se podrá disponer en todo momento para consultas de tipo TeóricoExperimental en Física por parte del alumnado matriculado/inscrito en la Plataforma Virtual de la Universidad de La Rioja. Así como poderlo utilizar para entablar debates sobre aspectos relacionados con la docencia impartida y/o cuestiones afines vinculadas con la investigación y el desarrollo científico-tecnológico.

* Y, ante todo, existe una muy apreciable mejora cualitativa y cuantitativa en el proceso de aprendizaje Teórico-Experimental en Física por parte de los alumnos, así como de sus resultados académicos. Es muy satisfactorio para el profesor, recibir un feedback tan positivo por parte de la mayoría del alumnado (>80\%), para el cual, este proyecto innovador crea hábitos muy positivos en la preparación previa del trabajo experimental en el laboratorio, sobre todo, porque son usuarios habituales de recursos multimedia. De hecho, ese porcentaje está en consonancia con la mejora experimentada en sus resultados académicos, en los cuales se producen aumentos en sus notas de prácticas en laboratorio de forma significativa: Notable (16\%) y Sobresaliente (43\%). 


\section{Conclusiones}

Las conclusiones que pueden extraerse de los resultados obtenidos a lo largo del desarrollo de este proyecto de innovación docente confirman un potencial real de la utilización racional de las TIC y la MFL. En un proceso sólido y fiable de aprendizaje TeóricoExperimental de la Física de los Sistemas Naturales y Artificiales $(\mathrm{I}+\mathrm{D}+\mathrm{i})$, y con la inestimable ayuda de un EV-3.0.

Una óptima preparación previa del trabajo teórico-experimental que va a desarrollar el GL mediante la MFL, así como la puesta en común de este proceso a través del mencionado EV-3.0, hacen de dicha metodología una herramienta muy interesante para obtener una mejora, tanto en el aprendizaje consolidado de la Física como los resultados académicos.

Por otra parte, su grabación en soporte audiovisual requiere una claridad de ideas sobre el proceso experimental tanto en su realización como en su descripción. Además, el intercambio a través del EV-3.0 de toda esta información multimedia, mejora sustancialmente el análisis de los procesos experimentales, los resultados obtenidos a partir de ellos y el debate constructivo entre alumnos y entre alumnos-profesor, generando un rico caldo de cultivo que alimenta un progreso científico-tecnológico sólido y transparente.

Sobra decir que todo el proceso está embebido en una praxis en torno a las TIC, que mejora las habilidades del alumnado respecto a todo aquello relacionado con esta nueva realidad virtual que ya está instalada entre nosotros.

\section{Referencias}

ALBA J., TORREGROSA C. y DEL REY R. (2015) Aprendizaje basado en proyectos: Primera experiencia en la asignatura de Física del Grado en Ingeniería de Telecomunicación, Sonido e Imagen. Universitat Politècnica de València Congreso IN-RED (2015).

APARICIO, F., GONZALEZ, R. M. y SOBREVILA, M. A. (2005). Formación de Ingenieros. Objetivos, métodos y estrategias. Instituto de Ciencias de la Educación, UPM.

BERNERS-LEE, T., HENDLER, J. y LASSILA, O. (2001). The Semantic Web: A new form of Web content that is meaningful to computers will unleash a revolution of new possibilities. Scientific American, Vol. 284: pp. 35-43.

BOLONIA. (2009). The Bologna Process 2020 - The European Higher Education Area in the new decade. Communiqué of the Conference of European Ministers Responsible for Higher Education.

CALVO, I., LOPEZ-GUEDE, J.M. y ZULUETA, E. (2010). Aplicando la metodología Project Based Learning en la docencia de Ingeniería Técnica en Informática de Gestión, Revista de Formación e Innovación Educativa Universitaria. Vol. 3, No 4, 166-181. 
CURY, A. (2007). Padres Brillantes, Maestros Fascinantes. Brasil, Planeta. ISBN: 9788408063605.

DESLAURIERS, L., SCHELEW, E. y WIEMAN C.. (2011). Improved Learning in a LargeEnrollment Physics Class, Science, Vol. 332, pp. 862-864. DOI: 10.1126/science.1201783.

DIAZ M. (2007) fq-experimentos. Experimentos caseros de Física y Química. <http://fq.experimentos.blogspot .com.es/> [Consulta: 29 de mayo de 2017]

FERRER C. (2012) Demostraciones experimentales de Física para el aula. <http://fisicademos.blogs.uv.es/?page_id=29> [Consulta: 12 de marzo de 2018]

FREEMAN, S., EDDY, S. L., MCDONOUGH, M., SMITH M. K., OKOROAFOR N., JORDT, H. y WENDEROTH, M. P. (2014). Active learning increases student performance in science, engineering, and mathematics, Proc. Natl. Acad. Sci. USA, Vol. 111, pp. 8410-8415.

GONZÁLEZ, J. y WAGENAAR, R. (2003): Tuning Educational Structures in Europe. Informe Final - Proyecto Piloto, Fase 1, Bilbao, Universidad de Deusto.

GONZÁLEZ MARIÑO, J. C. (2008). TIC y la transformación de la práctica educativa en el contexto de las sociedades del conocimiento. Revista de Universidad y Sociedad del Conocimiento (RUSC). Vol. 5, n. ${ }^{\circ} 2$.

GRANADOS, J. (2011). The Challenges of Higher Education in the 21st Century, GUNi Newsletter, 5/11. (http://www.guninetwork.org/articles/challenges-higher-education-21st-century).

KARPICKE, J. D. y BLUNT, J. R. (2011). Retrieval Practice Produces More Learning than Elaborative Studying with Concept Mapping, Science, Vol. 331, pp. 772-775. DOI: 10.1126/science.1199327.

MAZUR, E. (1997). Peer Instruction: A User's Manual. New York: Prentice Hall Series in Educational Innovation.

MORA MORA H., AZORÍN LÓPEZ, J., JIMENO MORENILLA, A., SÁNCHEZ ROMERO, J. L., PUJOL LÓPEZ, F., GARCÍA RODRÍGUEZ, J., SERRA PÉREZ, J. A., MORELL GIMÉNEZ, V., RIVES PÉREZ, M. F., SAVAL CALVO, M., GARCÍA GARCÍA, A. y ORTS ESCOLANO, S. (2016). Nuevas tendencias web 3.0 para la mejora de los procesos docencia-aprendizaje. Innovaciones metodológicas en docencia universitaria: resultados de investigación (pp.1543-1558). Alicante: Universidad de Alicante, Vicerrectorado de Estudios, Formación y Calidad, Instituto de Ciencias de la Educación.

MORA MORA H., SIGNES PONT, M. T., DE MIGUEL CASADO, G. y GILART IGLESIAS, V. (2015). Management of social networks in the educational process, Computers in Human Behavior, Vol. 51, Part B, pp. 890-895. doi:10.1016/j.chb.2014.11.010.

NACER, H. y AISSANI, D. (2014). Semantic web services: Standards, applications, challenges and solutions, Journal of Network and Computer Applications, Vol. 44, pp. 134-151. DOI: 10.1016/j.jnca.2014.04.015.

ORTEGA CARRILLO, J. A.. (2004). Redes de aprendizaje y curriculum intercultural. Actas del XIII Congreso Nacional y II Iberoamericano de Pedagogía. Ed. Sociedad Española de Pedagogía. Valencia. 
PRIETO MARTÍN, A. (2017-03-12). Decálogo de innovación metodológica para que los alumnos aprendan más y mejor en las asignaturas universitarias. Blog Profesor 3.0. http://profesor3punto0.blogspot.com.es/2015/12/decalogo-de-innovacion-metodologica.html.

SALINAS, J. (2004). Innovación docente y uso de las TIC en la enseñanza universitaria. Revista Universidad y Sociedad del Conocimiento. Vol. $1, \mathrm{n} .^{\circ} 1$.

SILVA, J. M., RAHMAN, A. S. y EL SADDIK, A. (2008). Web 3.0: a vision for bridging the gap between real and virtual. Paper presented at the 1st ACM international workshop on Communicability design and evaluation in cultural and ecological multimedia system, Vancouver British Columbia, Canada.

VILAPLANA, R. GOMIS O. y HYDER A. (2011) "Working in terms of competences: activities designed using active methodologies”. Safeeullah Soomro New Achievements in Technology, Education and Development. Croatia: In-Tech. Cap. 22, Pags: 352-372.

W3C. (2001). W3C Semantic Web Activity. URL: http://www.w3.org/2001/sw/, Acceso: mayo 2016. 